\title{
Modelagem no Ensino/Aprendizagem de Física e os Novos Parâmetros Curriculares Nacionais para o Ensino Médio
}

Modelling in teaching/learning of physics and the new Brazilian high school curricular parameters

\author{
E. A. Veit \\ Instituto de Física, Universidade Federal do Rio Grande do Sul \\ Cx. P. 15051, 91051-970, Porto Alegre, RS, Brasil \\ E-mail: eav@if.ufrgs.br \\ V. D. Teodoro \\ Faculdade de Ciências e Tecnologia, Universidade Nova de Lisboa \\ 2825 Campus de Caparica, Portugal \\ E-mail:vdt@mail.fct.unl.pt
}

Recebido em 28 de novembro, 2001. Aceito em 3 de janeiro, 2002.

\begin{abstract}
Discute-se a importância da modelagem no ensino/aprendizagem de Física em conexão com os novos parâmetros curriculares nacionais para o ensino médio (PCNEM). Apresentam-se as características essenciais do software Modellus, concebido especialmente para modelagem em Ciências Físicas e Matemática sob uma visão de ensino que enfatiza, no processo de aprendizagem, a exploração e a criação de múltiplas representações de fenômenos físicos e de objetos matemáticos.
\end{abstract}

The relevance of modelling in the teaching/learning of physics is discussed in connection to the new Brazilian high school curricular parameters. The discussion introduces the main features of the software Modellus, which has been especially conceived for modelling in Physical Science and Mathematics, based on a point of view that emphasizes in the learning process the creation and exploration of multiple representations for physical phenomena and mathematical objects.

\section{Introdução}

A utilização das novas tecnologias de informação e comunicação no ensino, especificamente a Internet e softwares educacionais, tem sido alvo de grande interesse, tanto para o ensino presencial quanto para o ensino aberto e a distância. Este não é um fenômeno nacional; pelo contrário, a maior parte dos países desenvolvidos e em desenvolvimento tem programas específicos para promover essa utilização. Também não parece ser um fenômeno nacional - pois em Portugal e em outros países europeus também é assim - que a política de incentivo tende a privilegiar a Internet como suporte de ensino. Embora ferramenta indispensável para o ensino a distância e que em muito enriquece o ensino presencial, há que se tomar cuidado para que o uso do computador não se restrinja a uma máquina de fornecer informação, como aconteceu com grande parte dos programas tutoriais do passado. Como a maioria dos educadores, defendemos o uso do computador como uma ferramenta para auxiliar a construção do conhecimento $[1,2]$, perspectiva esta que teve como expoente Papert [3]. Esta também é (felizmente) a perspectiva que embasa a quase totalidade das publicações [4] na Revista Brasileira de Ensino de Física e no Caderno Catarinense de Ensino de Física.

Os trabalhos já publicados nestas revistas podem ser classificados em três grandes categorias nas quais são discutidos: i) princípios e idéias gerais sobre a possibilidade de uso de novas tecnologias no ensino de Física; ii) o uso de um determinado software e seu entorno docente; iii) aquisição automática de dados em laboratórios didáticos de Física. Nenhum deles dá devida ênfase à modelagem como uma ferramenta computacional das mais preciosas para o ensino de Física sob uma perspectiva mais geral, conquanto haja bons pro- 
jetos que se valem da modelagem $[5,6]$. Tampouco há dentre as publicações que se dedicam à utilização de algum software em particular [7-10], uma que ilustre a potencialidade do Modellus [11], que é um software educacional desenvolvido especialmente para a modelagem no ensino de Ciências e Matemática e que tem sido amplamente usado em diversos projetos tanto nacionais quanto internacionais [12-14].

A presente publicação visa preencher parte desta lacuna, discutindo a importância da modelagem computacional no ensino/aprendizagem de Física - em conexão com os novos parâmetros curriculares nacionais para o ensino médio [15] - e apresentando o Modellus como uma ferramenta computacional que através $d a$ experimentação conceitual favorece a aprendizagem do jogo da modelagem [16]. Nosso enfoque se concentra em modelos conceituais, que são versões didáticas de modelos físicos e, especialmente, nos modelos matemáticos subjacentes, não nos modelos mentais e nas representações internas [17].

\section{Software de modelagem no ensino/aprendizagem de Física e os novos parâmetros curriculares nacionais para o ensino médio}

A Ciência é um processo de representação do Mundo, sempre sujeito a reformulação. A linguagem matemática desempenha um papel fundamental nesta representação, que não pode ser confundida com explicação. Na realidade, o discurso científico tem mais a ver com representações do que com explicações. Por exemplo, a lei da gravitação universal de Newton é uma forma de representar, através de um modelo matemático, a interação entre corpos celestes. Nada nos diz acerca do que é gravitação. O poder da linguagem matemática resulta, pois, não da sua capacidade de explicação, mas da sua capacidade de representação, de descrição do processo natural. Isto é, utilizando-se equações, é possível reproduzir no papel (no caso de Newton, que não tinha computador, mas paciência para realizar inúmeros cálculos repetitivos...) ou no computador o que se passa no céu (com certo grau de aproximação)!

Utilizamos a palavra modelagem $^{1}$ no sentido de um processo de representação. Um modelo é uma representação simplificada de um sistema, mantendo apenas as suas características essenciais. Um modelo matemático, que é uma forma específica de representação, se vale de objetos matemáticos, como são as funções, os vetores, as figuras geométricas. De particular interesse em Física são os modelos de sistemas dinâmicos, isto é, modelos que estabelecem alguma relação matemática entre quantidades físicas e o tempo, considerado como uma variável independente. Estes são os modelos em que estamos particularmente interessados neste artigo, pois a maior parte dos conteúdos de Física da escola de ensino médio e universitário está ancorada neste tipo de modelo, ainda que muitas vezes isto não seja transparente ao estudante.

Na prática, Física representa para o estudante, na maior parte das vezes, uma disciplina muito difícil, em que é preciso decorar fórmulas cuja origem e finalidade são desconhecidas. A introdução de modelagem no processo ensino/aprendizagem tende a desmitificar esta imagem da Física, possibilitando uma melhor compreensão do seu conteúdo e contribuindo para o desenvolvimento cognitivo em geral, pois modelagem facilita a construção de relações e significados, favorecendo a aprendizagem construtivista, podendo, também [18]:

- elevar o nível do processo cognitivo, exigindo que os estudantes pensem num nível mais elevado, generalizando conceitos e relações;

- exigir que os estudantes definam suas idéias mais precisamente;

- propiciar oportunidades para que os estudantes testem seus próprios modelos cognitivos, detectem e corrijam inconsistências.

Uma das mais importantes características dos programas de modelagem é a possibilidade de construir múltiplas representações de uma mesma situação. De certo modo, compreender um modelo e o respectivo fenômeno é ser capaz de construir múltiplas representações e "navegar", entre uma e outra [19].

Mas aprender não é, apenas, um processo de construção pessoal, desconectado das interações sociais e das ferramentas no processo de construção de conhecimento [20,21]. Sob este ponto de vista, softwares de modelagem são ferramentas da maior valia no ensino/aprendizagem das Ciências da Natureza $e$ da Matemática do século XXI, pois a compreensão do saber científico passa pelo exercício de modelagem e estes softwares costumam ser tais que facilitam estudos exploratórios individuais, assim como podem servir como elementos motivadores para o trabalho coletivo.

Dentre os princípios norteadores gerais estabelecidos pelas Diretrizes Curriculares para o Ensino Médio [22] destacam-se a produção de um conhecimento efetivo, não somente propedêutico, que leve ao desenvolvimento de competências e habilidades específicas para cada disciplina, integradas pela interdisciplinaridade e se valendo da contextualização. A organização do currículo em três grandes áreas (Linguagens e Códigos,

\footnotetext{
${ }^{1}$ Nos agrada mais o termo modelação, usado em Portugal, mas parece não ter aceitação entre os físicos brasileiros.
} 
Ciências da Natureza e Matemática e Ciências Humanas, cada uma delas acompanhada de suas Tecnologias) pretende conferir unidade ao ensino das diferentes disciplinas da área, orientando o trabalho integrado dos professores das respectivas áreas, sem dispensar uma articulação das áreas entre si.

Destaque-se que as grandes áreas incluem as suas tecnologias, dentre elas os computadores que revolucionaram a tal ponto a investigação científica que "hoje a computação científica pode ser considerada como uma terceira metodologia da ciência, paralelamente aos paradigmas mais estabelecidos da ciência teórica e experimental" [23]. A utilização das novas tecnologias na educação está muito defasada em relação ao seu uso científico - também em nível internacional - mas o que se espera, e se preconiza nos parâmetros curriculares nacionais para o ensino médio (PCNEM) é que as tecnologias específicas de cada área venham a ser incorporadas no seu processo ensino/aprendizagem.

Na área de Ciências da Natureza, Matemática $e$ suas Tecnologias, na qual a disciplina de Física está inserida, entende-se que "A aprendizagem de concepções científicas atualizadas do mundo físico e natural e o desenvolvimento de estratégias de trabalho centradas na solução de problemas são finalidade da área, de forma a aproximar o educando do trabalho de investigação científica e tecnológica, como atividade institucionalizadas de produção de bens e serviços." [15, p. 33]

Pergunta-se: como aproximar o educando do trabalho de investigação científica em Física e Matemática sem trabalhar com modelos?

Nos PCNEM, os objetivos curriculares são focados em competências e habilidades a serem atingidas pelos estudantes nas diferentes disciplinas, ao invés de focados nos conteúdos específicos cobertos por essas disciplinas. Esta perspectiva altera completamente a organização curricular, pois passam a ser as competências que orientam a seleção e o ordenamento de conteúdos, com seus respectivos tempos e espaços curriculares. Justamente por isso, estamos vivenciando um processo de reorganização curricular de nossos cursos de Licenciatura em Física, a fim de que satisfaçam as Diretrizes Curriculares para a Formação Inicial de Professores da Educação Básica, em Cursos de Nível Superior [24], preparando o professor que deverá trabalhar no nível médio à luz dos novos parâmetros curriculares.

Esta discussão sobre a renovação de currículo, assim como as anteriores, também não é um fenômeno apenas nacional [25]. Ela tem ocorrido em diversos países nos últimos quinze anos, devendo-se, especialmente, ao progresso obtido na investigação educacional, particularmente com a identificação de concepções alternativas e as dificuldades de mudança conceitual. Ao contrário das reformas das décadas de 60 e 70 [26], as mais recentes deram mais importância às dificuldades de conhecimentos sobre os processos de aprendizagem [27]. Um sucinto histórico das reformas curriculares brasileiras na área de Matemática e Ciências da Natureza consta na seção rumos e desafios dos PCNEM [15, p. 261 a 263]. Nas reformas recentes é marcante a importância dada a uma visão mais integrada, desde a aprendizagem da comunicação escrita e oral, até à necessidade de aprendizagem em contextos interdisciplinares e às conexões entre as abordagens das diversas ciências, como proposto nos PCNEM. Outra característica é uma educação com "maior ambição formativa, tanto em termos da natureza das informações tratadas, dos procedimentos e atitudes envolvidas, como em termos das habilidades, competências e dos valores desenvolvidos." [op. cit. p. 207]

Dentre as competências e habilidades a serem desenvolvidas na área de Ciências da Natureza, Matemática e suas Tecnologias, há diversas que seriam diretamente beneficiadas pelo uso da modelagem no processo de ensino/aprendizagem, especialmente se, para tanto, se utilizar uma ferramenta computacional como o Modellus, que dispensa qualquer linguagem de programação. Aqui, nos concentramos especificamente nas competências e habilidades a serem desenvolvidas em Física e destacamos as que seguem [op. cit. p. 237]:

- Compreender enunciados que envolvam códigos e símbolos físicos.

- Utilizar e compreender tabelas, gráficos e relações matemáticas gráficas para a expressão do saber físico. Ser capaz de discriminar e traduzir as linguagens matemática e discursiva entre si.

- Expressar-se corretamente utilizando a linguagem física adequada e elementos de sua representação simbólica. Apresentar de forma clara e objetiva o conhecimento apreendido, através de tal linguagem.

- Elaborar sínteses ou esquemas estruturados dos temas físicos trabalhados.

- Desenvolver a capacidade de investigação física. Classificar, organizar, sistematizar. Identificar regularidades. Observar, estimar ordens de grandeza, compreender o conceito de medir, fazer hipóteses, testar.

- Conhecer e utilizar conceitos físicos. Relacionar grandezas, quantificar, identificar parâmetros relevantes. Compreender e utilizar leis e teorias físicas.

- Construir e investigar situações-problema, identificar a situação física, utilizar modelos físicos, generalizar de uma a outra situação, prever, avaliar, analisar previsões.

"É preciso mudar convicções equivocadas, culturalmente difundidas em toda a sociedade, de que os alunos são os pacientes, de que os agentes são os professores e de que a escola estabelece simplesmente o cenário do processo de ensino." [op. cit. p. 263] 
"Se há uma unanimidade, pelo menos no plano dos conceitos entre educadores para as Ciências e a Matemática, é quanto à necessidade de se adotarem métodos de aprendizado ativo e interativo." [op. cit. p. 266]

É esta visão sobre o processo de aprendizagem que fundamenta a utilização de softwares de caráter exploratório como o Modellus e os de geometria dinâmica (Sketchpad, Cabri, Cinderella, Supposer,...). Nesta perspectiva, o computador é visto como uma ferramenta para auxiliar a construção do conhecimento, em contraposição à outra perspectiva dominante, em que os computadores são utilizados como máquinas de informação, como é o caso da quase totalidade das páginas da Internet. (Embora mereça ser registrado o valor indiscutível de alguns destes materiais de divulgação [28].)

\section{Modellus: essenciais}

Modellus é uma ferramenta cognitiva para auxiliar a internalização de conhecimento simbólico, preferencialmente em contexto de atividades de grupo e de classe, em que a discussão, a conjetura e o teste de idéias são atividades dominantes, em oposição ao ensino direto por parte do professor. Isto não significa, no entanto, que os estudantes reinventam o conhecimento quando constroem ou exploram modelos com o Modellus. De fato, ninguém pode aprender explorando sem conhecimento relevante sobre o campo de exploração. A aquisição de conhecimentos e capacidades não é um processo completamente claro e definido no tempo e no espaço. É demorado, contextual, dependente de estruturas cognitivas e conhecimento prévio. E, essencialmente, é um processo de familiarização com novas idéias e representações (como afirmaram muitos dos mais notáveis criadores científicos, como Newton, Planck, Feynman).

Modellus, como outras ferramentas computacionais, permite ao usuário fazer e refazer representações, explorando-as sobre as mais diversas perspectivas. Deste modo, facilita a familiarização com essas representações, criando de certo modo uma intimidade entre aprendiz e representação, intimidade essa que muito dificilmente resulta da simples observação ocasional de equações e representações feitas pelo professor ou apresentadas nos livros. Essa intimidade, por outro lado, é fundamental para a reificação dos objetos formais, algo que, de acordo com Roitman [29], é imprescindível no desenvolvimento do pensamento científico.
Antes do advento dos computadores, construir e explorar modelos matemáticos exigia grande capacidade de abstração. Por exemplo, a simples representação de um movimento acelerado por uma função do segundo grau, requer do aprendiz a utilização, e portanto a compreensão de uma função do tipo:

$$
x=\frac{1}{2} a_{x} t^{2}
$$

Dispondo de uma ferramenta computacional, o processo de modelagem adquire contornos mais concretos, pois o aprendiz pode utilizar esta função em um contexto específico e explorar o significado dos seus parâmetros e da função em si. Especialmente, se a ferramenta computacional dispensar o conhecimento de uma linguagem especial de programação. Esta é uma das maiores virtudes do Modellus: a sintaxe de escrita é praticamente a mesma que se usa ao escrever um modelo no papel, tanto para funções quanto para equações diferenciais ordinárias. Assim, o usuário não precisa aprender nenhuma linguagem de programação, bastando conhecer o simbolismo matemático, como pode ser visualizado na janela Modelo apresentada na Fig. 1. (Caso não domine a linguagem matemática, Modellus poderá auxiliá-lo na sua compreensão. Tanto quanto é do nosso conhecimento, Modellus é o único software de autor que dispensa completamente qualquer linguagem ou metáfora de programação, ao contrário do que ocorre com excelentes ferramentas para modelagem, como o LOGO [30] e o STELLA [31] .)

Justamente por se valer da linguagem matemática usual é que Modellus se torna um excelente programa também para o ensino de Matemática, assim como em qualquer outra área do conhecimento que se valha de sistemas dinâmicos, como a Biologia e a Química.

O mapa cognitivo apresentado na Fig. 2 fornece uma idéia geral da concepção e das possibilidades de uso do Modellus, que passamos a descrever.

Modellus foi concebido como um software de modelagem, no qual o usuário pode facilmente escrever modelos matemáticos expressos como funções, equações diferenciais, equações a diferenças finitas ou derivadas. Tomemos, como exemplo, o modelo de um objeto considerado como uma partícula (objeto com massa mas sem dimensão), que se move unidimensionalmente com velocidade constante.

A distância percorrida pela partícula, $s$, pode ser expressa como uma função linear do tempo, t:

$$
s=v_{x} t
$$




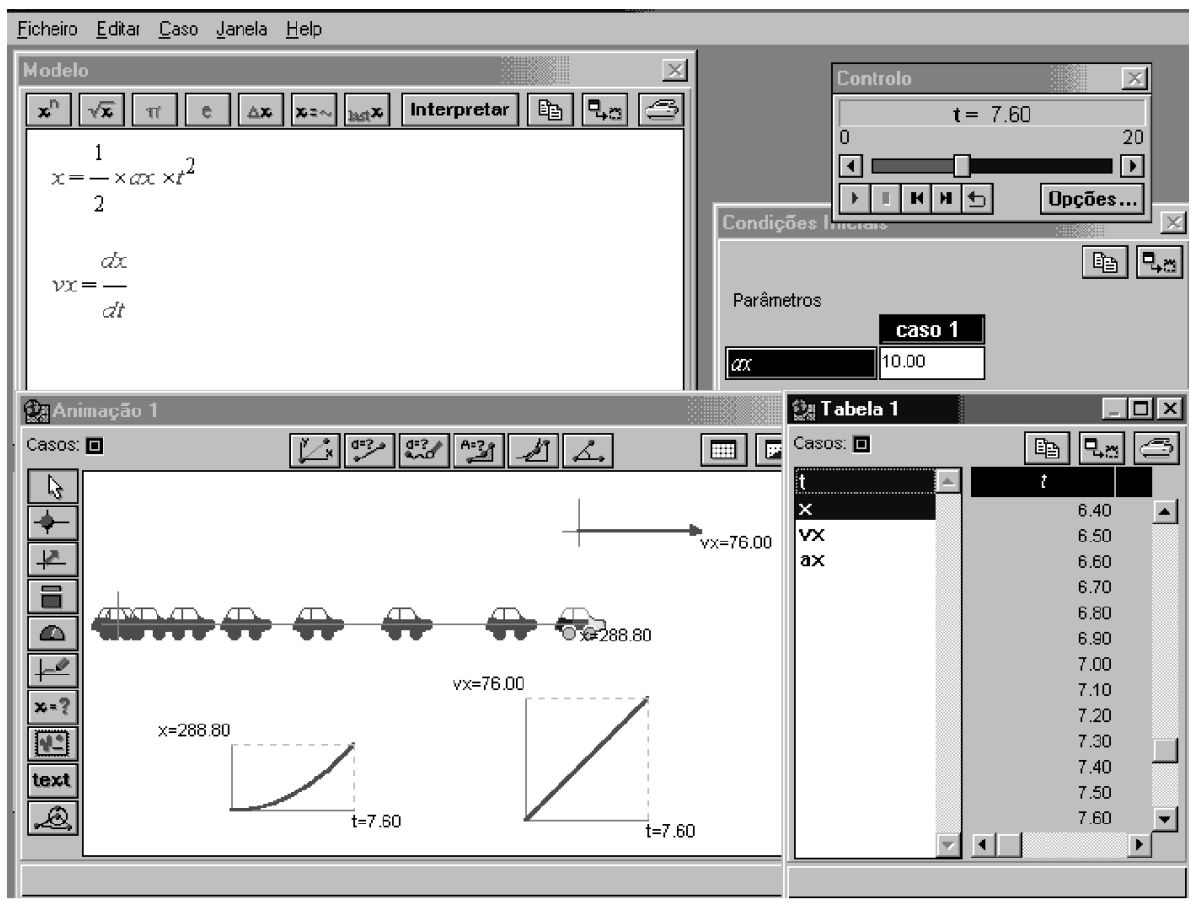

Figura 1: A sintaxe de escrita - na janela Modelo - é muito semelhante à linguagem matemática utilizada no quadro-negro.

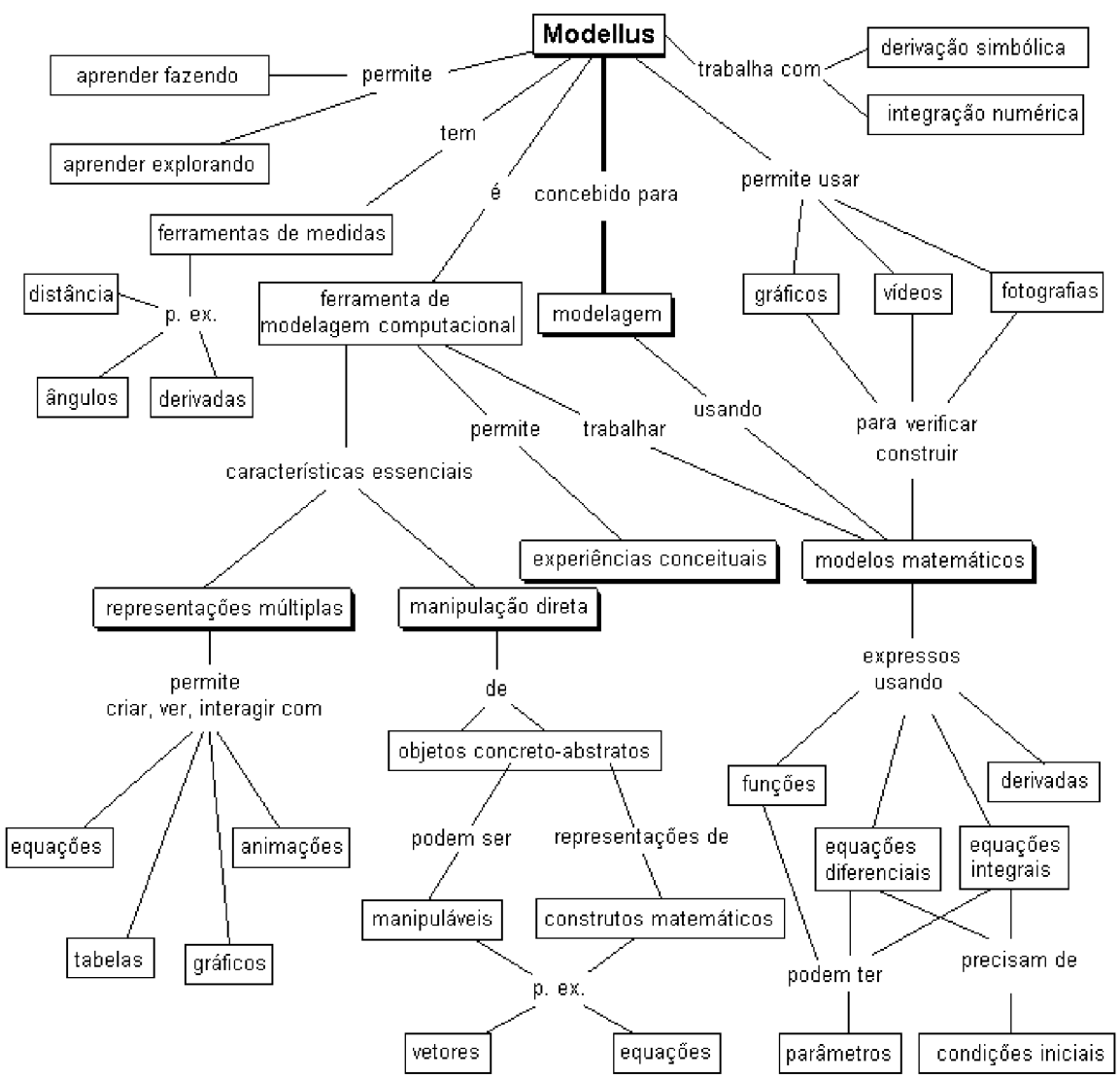

Figura 2. Mapa cognitivo sobre o Modellus. 
Um modelo equivalente pode ser construído representando a coordenada $x$, num determinado sistema de referência cuja orientação do eixo $O x$ coincide com a trajetória da partícula, como uma função linear do tempo:

$$
x=x_{0}+v_{x} t \quad .
$$

Nesta equação $x_{0}$ representa a coordenada $x$ da partícula no instante inicial $(t=0)$ e $v_{x}$ representa a componente escalar da velocidade na direção $O x$.

Ao acionar o botão Interpretar, Modellus imediatamente solicitará ao aprendiz que especifique valores para os parâmetros $x_{0}$ e $v_{x}$.

Esta mesma situação física pode ser modelada usando-se o conceito de taxa de variação. Como a taxa de variação da coordenada $-x, v_{x}$, é constante, tem-se:

$$
\frac{d x}{d t}=v_{x}
$$

Neste caso, ao interpretar o modelo, serão solicitadas as condições iniciais, indispensáveis para a solução do problema específico.

Estes três modelos são equivalentes e podem ser utilizados em diferentes estágios da aprendizagem. Os dois primeiros se valem de funções, enquanto o último é especificado por uma equação diferencial ordinária, com as necessárias condições iniciais que especificam a situação particular em estudo.

Um modelo para esta mesma situação física pode, ainda, ser escrito em termos de diferenças finitas. Neste caso, usa-se a notação usual em linguagem de computação, na qual o sinal de igual significa que o lado esquerdo da equação é substituído pelo lado direito. Então,

$$
x={ }_{\text {last }} x+\nu x \times \Delta t
$$

onde $\Delta t$ é um "pequeno" intervalo de tempo.

Alguns autores [32] e, mais recentemente, alguns projetos, como o do Institute of Physics do Reino Unido $^{2}$, enfatizam o uso de equações a diferenças finitas na introdução aos conceitos de Cálculo, como taxas de variação, derivadas e integrais, em um nível de escolaridade anterior ao universitário.

Na construção de modelos, o usuário pode se valer, também, da derivada de qualquer ordem de uma função de uma variável.

Sob o ponto de vista de cálculo, Modellus realiza: i) as integrações numericamente, valendo-se do Método de Runge-Kutta de $4^{a}$ ordem; ii) as derivações simbolicamente ou aproxima-as por taxas de variação; iii) cálculos em um modo iterativo.

Papert foi um dos primeiros autores a conjeturar que o computador poderia concretizar o formal, possibilitando que conhecimento que só pode ser acessado através de processo formal fosse atingido pela sucessão de processos concretos - facilitando, assim, a transição concreto-formal do pensamento. A importância da concretização do formal, sem perder a idéia da relevância do abstrato na construção do conhecimento científico, tem sido retomada por vários outros autores. Hebenstreit [33] considera que uma das principais potencialidades do computador é permitir a construção de objetos "concreto-abstratos": concretos porque podem ser diretamente manipulados no computador; abstratos porque são representações de idéias ou relações. Por exemplo, uma soma de dois vetores pode ser feita no Modellus tanto escrevendo-a como uma soma de suas componentes vetoriais, quanto manipulando diretamente na tela as setas que representam os vetores. Também os objetos e as quantidades físicas, associadas a cursores podem ser alteradas diretamente na tela, durante o transcorrer da animação.

À época em que Papert lançou estas idéias, as interfaces gráficas computacionais eram pouco desenvolvidas, e o objeto concreto criado foi uma tartaruga, que só podia ser movimentada por comandos de uma linguagem computacional - LOGO. Modelar com esta linguagem em Física não é tarefa trivial, ao alcance de estudantes que não têm maior aptidão e/ou interesse por ciências exatas, ou seja, para a maior parte dos estudantes do ensino médio. Ainda em 1995, Nickerson [34] assinalava que não era dada devida atenção à criação de programas onde os estudantes pudessem construir simulações facilmente. Pois a premissa básica na concepção do Modellus foi a de que na construção de um modelo o aprendiz deveria pensar como se estivesse usando papel e lápis, e que os objetos na tela fossem manipuláveis.

Tomemos, como exemplo, o movimento acelerado apresentado na Fig. 1. Na janela Modelo, estão indicadas as equações do modelo do movimento. A posição $x$ é definida explicitamente como função de $t$, e $v x$ (a componente da velocidade segundo o eixo $O x$ ) é definida como a derivada da posição em relação ao tempo. Ao parâmetro $a x$, a componente da aceleração no eixo $O x$, é atribuído o valor 10 unidades $\left(\mathrm{m} / \mathrm{s}^{2}\right.$, no sistema internacional de unidades), na janela Condições Iniciais. Uma vez construído o modelo, construiu-se uma $A n i$ mação e uma Tabela. Na tabela, representam-se os valores das diversas variáveis. $\mathrm{Na}$ animação, aparece um objeto que se move com aceleração, conforme pode ser visto pela representação estroboscópica (isto é, representação da posição em diversos instantes separados por iguais intervalos de tempo); um vetor que representa a velocidade, acompanhando o movimento do objeto; e dois gráficos, um da posição e outro da magnitude da velocidade.

\footnotetext{
${ }^{2}$ IoP project Physics 16-19: Projeto que visa revitalizar o ensino de Física para estudantes entre 16 e 19 anos (http://www.iop.org/IOP/AP).
} 
Este exemplo ilustra a possibilidade de construir múltiplas representações da mesma situação. Aqui o estudante visualiza - ou constrói - simultaneamente, as equações, a tabela, os gráficos e a animação (ou simulação) do movimento.

Estas são características esperadas pelos PCNEM no conhecimento em Física como exposto por Menezes $^{3}$ : "Por exemplo, tanto as linguagens específicas da Física, derivadas de modelagens do mundo macroscópico ou microscópico e instrumentais para certas representações abstratas de eventos e processos, como outras linguagens de que a física faz uso, a exemplo da matemática, como as expressões algébricas, os gráficos cartesianos ou representações estatísticas, umas e outras passam a ser parte dos objetivos formativos do aprendizado da física, não simples pré-requisitos que o professor deve esperar que o aluno tenha adquirido em outra disciplina ou em outra circunstância." [35]

Modellus possibilita, ainda, a avaliação da qualidade de um modelo na descrição de dados experimentais, quer disponíveis na forma de gráficos, quer de fotografias, quer de vídeos. Por exemplo, na Fig. 3 é feita uma comparação entre o movimento da água de um chafariz e o resultado obtido com um modelo de queda livre.
Nas suas últimas versões, Modellus dispõe, também, de instrumentos que permitem a medida de quantidades físicas representadas na forma de gráficos, vídeos e fotografias. Estes instrumentos de medida (de ângulo, distância, área, coordenadas, derivada) podem servir para determinar os parâmetros a serem usados num modelo que descreverá estes dados, assim como podem ser usados na coleta de informação. Por exemplo, usando-se o instrumento de medida de ângulo, pode-se determinar o ângulo de emergência de dois objetos que colidem.

Sob o ponto de vista educacional, Modellus possibilita tanto o aprender fazendo quanto o aprender explorando [36], já que tanto o aprendiz pode construir seus próprios modelos, de um modo muito direto, insistimos, se valendo do simbolismo matemático como usualmente é manuscrito, assim como pode explorar modelos feitos pelo professor ou por outros. No caso de modelos feitos por outros, há a possibilidade de manter a janela Modelo fechada - inclusive bloqueando-a com senha - de modo que o aluno não a possa visualizar. Assim, equações diferenciais e derivações podem ser utilizadas também no ensino médio, em modelos que se destinam à exploração.

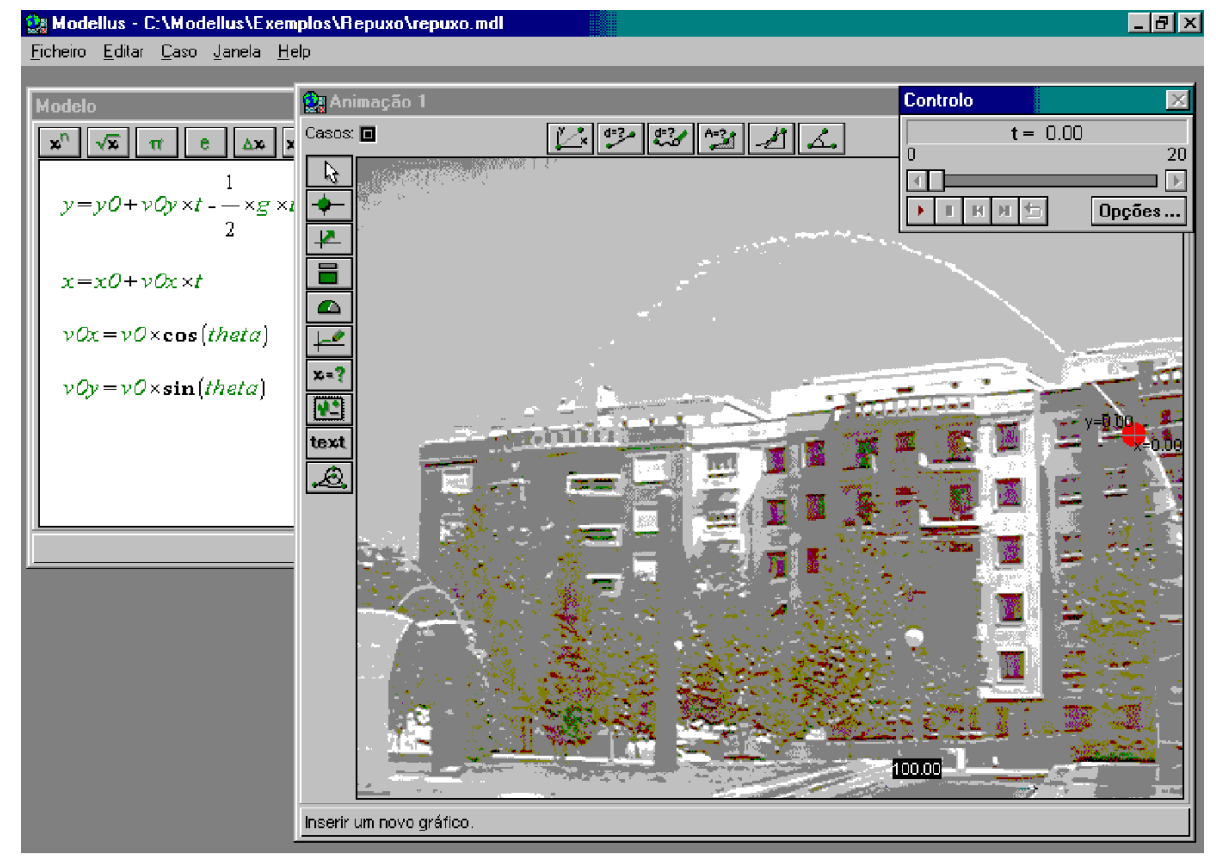

Figura 3. Avaliação de um modelo pela comparação entre a trajetória prevista pelo modelo e dados experimentais na forma de uma fotografia.

\footnotetext{
${ }^{3}$ O Prof. Luís Carlos Menezes coordenou a área de Ciências da Natureza e Matemática na elaboração dos Parâmetros Curriculares Nacionais para o Ensino Médio.
} 
Assim, especialmente porque Modellus permite: - construir e explorar múltiplas representações de modelos;

- analisar a qualidade dos modelos;

- reforçar o pensamento visual, sem memorização dos aspectos de representação formal através de equações e outros processos formais;

- abordar de uma forma integrada os fenômenos naturais, ou simplesmente representações formais;

- trabalhar individualmente e em classe, em que a discussão, a conjetura e o teste de idéias são atividades dominantes;

- ter sua utilização no ensino/aprendizagem de Física como ferramenta extremamente poderosa para o desenvolvimento de competências / habilidades preconizadas pelos PCNEM e destacadas na seção II.

\section{Comentários finais}

Neste trabalho, nos concentramos na importância da modelagem no ensino de Física em nível médio em conexão com os PCNEM. Claro está que modelagem é uma ferramenta valiosa também no ensino universitário $^{4}$. Em ambos os níveis, uma das motivações de sua utilização é a possibilidade de se tratar de problemas mais realísticos e mais atuais. Por exemplo, não há justificativa, nos dias atuais, para se limitar o estudo de movimentos próximo à superfície terrestre ao movimento de queda livre. Sob o ponto de vista conceitual, este problema apresenta grau de dificuldade semelhante ao da situação mais realística em que se considera resistência do ar ou ação de vento. Tradicionalmente, restringiu-se o estudo de fenômenos físicos a casos ideais em que há solução analítica, mesmo quando o estudante não é capaz de obtê-la, por exemplo, o período do pêndulo simples no ensino médio. Resulta que, em não sendo capaz de derivar a solução, só lhe resta "decorar a fórmula" (e decorar, também, que esta fórmula só vale para pequenas amplitudes, expressão esta de que, na maior parte das vezes, sequer sabe o significado). Defendemos que seria muito mais formativo e motivador estimular estudos exploratórios com um software de modelagem que permita fazer experiências conceituais e/ou construir modelos que descrevam dados. (Neste caso, nem há por que se limitar a pequenos ângulos, pois a solução numérica obtida vale para qualquer amplitude. Então, sim, fazer a discussão para pequenos ângulos e incluir resistência, ou força externa, discutir ressonância.) Apontamos para a possibilidade de se inserir novos tópicos, como fenômenos não-lineares e caos. Acreditamos que modelagem é um dos meios indispensáveis para uma mudança radical do ensino médio, no sentido sugerido por Moreira [25]:
"Física não dogmática, construtivista, para a cidadania, ênfase em modelos, situações reais, elementos próximos, práticos e vivenciais do aluno, do concreto para o abstrato, atualização de conteúdos, Física Contemporânea."

Não se trata, obviamente, da substituição do laboratório didático pela modelagem computacional. Tratase da sua complementação, de ampliar limites, de reforçar o aspecto construtivista da ciência e da aprendizagem, o pensamento científico - não a lógica indutiva. Também não se trata de investir esforços exclusivamente em modelagem deixando de considerar contribuições importantes advindas de outras vertentes como "História e Filosofia da Ciência" e "Física Contemporânea" [25] . Trata-se de agregar uma nova tecnologia que facilita o processo de aprendizagem, que contribui para o desenvolvimento cognitivo e propicia uma melhor compreensão da ciência e da tecnologia, também pelo estudante que não prosseguirá seus estudos.

A presença de novas tecnologias na educação, contudo, não garante uma mudança efetiva nos processos de ensino/aprendizagem: é indispensável o uso adequado destas tecnologias [38]. Neste sentido, são muito bem-vindas as iniciativas no sentido de criar material didático adicional, que dêem suporte ao uso de softwares educacionais, como o desenvolvido com a linguagem LOGO [5,6] e com o Modellus [12-14] que tem sido muito valioso para professores e estudantes que se motivam a utilizar estes softwares.Ainda assim, isto de per si não é suficiente, pois, via de regra, ainda temos limitado o uso de ferramentas computacionais a um número diminuto de oportunidades.

Como se observa nas reformas curriculares mais recentes, e preconizam os PCNEM em nível nacional, é preciso dar uma visão mais integrada dos currículos, desde a aprendizagem da comunicação oral, até à necessidade de aprendizagem em contextos interdisciplinares e às conexões entre as abordagens das diversas ciências.

A integração da Física com a Matemática e com suas tecnologias recebeu recentemente um novo impulso com o projeto do Institute of Physics do Reino Unido em que o Modellus é considerado como uma ferramenta que faz parte do curso [14]. Esse é, provavelmente, o primeiro projeto de ensino não superior em que a utilização de ferramentas computacionais - especificamente o $\mathrm{Mo}$ dellus e a planilha eletrônica - desempenha um papel essencial, quer para a exemplificação de situações quer para a aprendizagem da construção de modelos. Por exemplo, no início do segundo ano do curso (o último ano do ensino médio) o estudante constrói modelos utilizando funções e equações diferenciais. Este é um curso inovador nos tópicos abordados, como tratamento de imagens, instrumentação e sistemas de comunicação,

\footnotetext{
${ }^{4}$ Os documentos pertinentes neste caso seriam as Diretrizes Curriculares para a Formação Inicial de Professores da Educação Básica, em Cursos de Nível Superior [24] e as Diretrizes Curriculares para os Cursos de Graduação em Física [37], atualmente em análise no Conselho Nacional de Educação.
} 
com grande ênfase no caráter conceitual. Entendemos que o ensino baseado em excelentes livros didáticos existentes no mercado nacional, como o livro de Física do GREF [39], o Curso de Fúsica, de Alvarenga e Máximo [40], e Física, de Gaspar [41], precisaria ser enriquecido com a utilização de modelagem computacional e de outros novos recursos.

Numa perspectiva mais ampla, é necessária uma reflexão sistemática sobre o melhor processo de concretizar uma visão integrada dos conteúdos e sobre qual o papel das ferramentas computacionais nesta visão. Em relação ao Modellus, esta reflexão está em andamento no Brasil, em Portugal, na Inglaterra e em diversos outros países. Seria oportuna uma investigação sobre sua contribuição na compreensão de modelos físicos sob o referencial de modelos mentais [17]. Também é preciso que haja um esforço continuado no sentido de influenciar o desenvolvimento curricular e as práticas pedagógicas sobre o uso de tecnologia, sem jamais esquecer que, além da tecnologia de qualidade, do entorno docente, de práticas educacionais embasadas em pesquisas educacionais, há que se investir no professor, cuja resistência e dificuldade de aprendizagem nesta área podem ser bem maiores do que as do estudante.

\section{Agradecimentos}

Agradecemos aos professores M. A. Moreira e P. M. Mors pela leitura crítica deste manuscrito.

\section{Referências}

[1] CORTE, E. de; VERSCHAFFEL, L.; LOWYCK, J. Computers and learning. In: HUSÉN, T. N. (Eds.). Education: the complete encyclopedia. Oxford: Pergamon Press, 1998. 1 CD

[2] TAYLOR, R. P. (Ed.) The computer in the school: tutor, tool, tutee. New York: Teachers' College Press, 1980.

[3] PAPERT, S. Mindstorms: children, computers and powerful ideas. New York: Basic Books, 1980.

[4] VEIT, E. A. Disponível em: $<$ http://www.if.ufrgs.br/cref/ntef/publica.html>. Acesso em: 30 out. 2001.

[5] AGUIAR, C. E. Informática no ensino de ciências. Disponível em: $<$ http://www.if.ufrj.br/ carlos/infoenci/infoenci.html $>$. Acesso em: 30 out. 2001.

[6] Programa Educ@r para aluno de ensino médio. São Carlos: Universidade de São Paulo. Disponível em: $<$ http://educar.sc.usp.br/fisica/>. Acesso em: 30 out. 2001.

[7] SAnTOS, G.; OTERO, M. R.; FAnARO, M. A. Como usar software de simulación en clases de física? Cad. Catarinense Ens. Fis., Florianópolis, v. 17, n. 1, p. 5066, abr. 2000.
[8] SANTOS, A. C. K. dos; CHO, Y.; ARAUJO, I. S.; GONÇALVES, G. P. Algumas possibilidades de utilização dos princípios de sistemas Forrester em tópicos de física, através da ferramenta de modelagem quantitativa STELLA Cad. Catarinense Ens. Fis., Florianópolis, v. 17 , n. 1, p. 81-95, abr. 2000.

[9] RESENDE, F. Desenvolvimento e avaliação de um sistema hipermídia para facilitar a reestruturação conceitual em mecânica básica, Cad. Catarinense Ens. Fis., Florianópolis, v. 18, n. 2, p. 197-213, ago. 2001.

[10] CAMILETTI, G. e FERRACIOLI, L. A utilização da modelagem computacional quantitativa no aprendizado exploratório de Física, Cad. Catarinense Ens. Fis., Florianópolis, v. 18, n. 2, p. 214-228, ago. 2001.

[11] TEODORO, V. D. From formulae to conceptual expriments: interactive modelling in the physical sciences and in mathematics. In: INTERNATIONAL CoLos CONFERENCE NEW NETWORK-BASED MEDIA IN EDUCATION, 1998, Maribor, Eslovênia. [S.l.: s.n.], 1998. p. 13-22; Modellus: experiments with mathematical models. Disponível em:

$<$ http://phoenix.sce.fct.unl.pt/modellus/>. Acesso em: 20 out. 2001.

[12] TEODORO, V. D.; VIEIRA, J. P. D.; CLÉRIGO, F. C. Modellus 2.01: interactive modelling with mathematics. Monte Caparica: Faculdade de Ciência e Tecnologia Universidade Nova de Lisboa, 2000.

[13] VEIT, E. A.; MORS, P. M. Física geral universitária: mecânica. Instituto de Física - UFRGS, 1999.

[14] LAWRENCE, I.; WHITEHOUSE, M. (Eds.) Advancing physics. Bristol: Institute of Physics, 2000. 1 CD. Teacher's version.

[15] Brasil. Ministério da Educação, Secretaria de Educação Média e Tecnológica. Parâmetros curriculares nacionais: ensino médio. Brasília: MEC, 1999.

[16] HALLOUN, I. Schematic modeling for meaningful learning of physics. J. Res. Sci. Teach., New York, v. 33, n. 9, p. 1019-1041, nov. 1996.

[17] GRECA, I. M.; MOREIRA, M. A. Mental, physical, and mathematical models in the teaching and learning of physics, Science Education, a v. 86, p. 106-121, 2002.

[18] WEBB, M.; HASSEN, D. Opportunities for computer based modelling and simulation in secondary education. In: LOVIS, F.; TAGG, E. D. (Eds.). Computers in education. Amsterdam: North-Holland, 1988.

[19] EDUCATIONAL TECHNOLOGY CENTER. Making sense of the future. Cambridge: Harvard Graduate Shool Education, 1988.

[20] VIGOTSKY, L. S. Mind in society: the development of higher psychological processes. Cambridge: Harvard University Press, 1978.

[21] RESNICK, L. B.; COLLINS, A. Cognition and learning. In: HUSÉN, T. (Eds.) Education: The complete encyclopedia. Oxford: Pergamon Press, 1998. 1 CD.

[22] CÂMARA DE ENSINO BÁSICO DO CONSELHO NACIONAL DE EDUCAÇÃO. Resolução CEB n. 3, 26 jun. 1998. Disponível em:

<http://www.mec.gov.br/semtec/revista/dircur.shtm >. Acesso em 30 out. 2001. 
[23] NATIONAL RESEARCH COUNCIL. Everybody counts. Washington: National Academy of Sciences, 1989. p. 36.

[24] CONSELHO NACIONAL DE EDUCAÇÃO. Parecer CNE/CP 027/2001 e CNE/CP 028/2001, 2 out. 2001. Disponível em: $<$ http://www.mec.gov.br/cne/parecer.shtm\#2001>. Acesso em: 30 out. 2001.

[25] MOREIRA, M. A. Ensino de física no Brasil: retrospectiva e perspectiva. Rev. Bras. Ens. Fis., São Paulo, v. 22 , n. 1, p. 94-99, mar. 2000.

[26] RAIZEN, S. A., The reform of science education in the U.S.A. Déjà vu or de novo? Studies in Science Education, Driffield, v. 19, p. 1-41, 1991.

[27] EYLON, B. S. Physical Sciences: secondary school programs. In: HUSÉN, T. (Eds.). Education: the complete encyclopedia. Oxford: Pergamon Press, 1998. 1 CD.

[28] MOREIRA, J. E. Disponível em: $<$ http://www.fisica.ufc.br/>. Acesso em: 30 out. 2001.

[29] ROITMAN, J. A mathematician looks at national standards, Teachers College Record, 1 (fall), p. 22-44, 1998.

[30] LOGO FOUNDATION. Disponível em: $<$ http://el.www.media.mit.edu/logo-foundation/>. Acesso em: 30 out. 2001.

[31] RICHMOND, B. et al. An academic user's guide to STELLA. Lyme: High Performance System, 1987.

[32] OGBORN, J. A microcomputer modelling system and the teaching of problem structure. Recherche en didactique de la physique. Paris: Éditions du CNRS, 1984.
[33] HEBENSTREIT, J. Simulation et pédagogie, une rencontre du troisième type. Gif Sur Yvette: École Superieure d'Electricité, 1987.

[34] NICKERSON, R. S. Can technology help teach for understanding? In: PERKINS, D. N. (Eds.). Software goes to school. New York: Oxford University Press, 1995.

[35] MENEZES, L. C. de. Uma física para o novo ensino médio. Física na Escola, São Paulo, v. 1, n. 1, p. 6-8, out. 2000.

[36] BLISS, J.; OGBORN, J. Tool for exploratory learning. Oxford, v. 5, n. 1, p. 35-50, Mar. 1989.

[37] MINISTÉRIO DA EDUCAÇÃO. Diretrizes curriculares para os cursos de graduação. Disponível em: <http://www.mec.gov.br/Sesu/diretriz.shtm\#diretrizes $>$. Acesso em: 30 out. 2001.

[38] BRANSFORD, J.; BROWN A.; COCKING, R. How people learn: brain, mind, experience and school. Washington: National Academy Press, 2000.

[39] GREF. Física. São Paulo: Editora da Universidade de São Paulo, 1993. 3v.

[40] LUZ, A. M. R. da; AlvareS, B. A. Curso de Física. São Paulo: Scipione, 1997. 3 v.

[41] GASPAR, A. Física. São Paulo: Editora Ática, 2000. 3 v. 